\title{
Novel postural control algorithm for control of multifunctional myoelectric prosthetic hands
}

\author{
Jacob L. Segil, PhD; ${ }^{*}$ Richard F. ff. Weir, $\mathbf{P h D}^{\mathbf{2}}$ \\ ${ }^{1}$ Department of Mechanical Engineering, University of Colorado at Boulder, Boulder, CO; ${ }^{2}$ Biomechatronics Develop- \\ ment Laboratory, Department of Veterans Affairs Eastern Colorado Healthcare System, Denver, CO; and Department \\ of Bioengineering, College of Engineering and Applied Science, University of Colorado Denver | Anschutz Medical \\ Campus, Denver, $C O$
}

\begin{abstract}
The myoelectric controller (MEC) remains a technological bottleneck in the development of multifunctional prosthetic hands. Current MECs require physiologically inappropriate commands to indicate intent and lack effectiveness in a clinical setting. Postural control schemes use surface electromyography signals to drive a cursor in a continuous twodimensional domain that is then transformed into a hand posture. Here, we present a novel algorithm for a postural controller and test the efficacy of the system during two experiments with 11 total subjects. In the first experiment, we found that performance increased when a velocity cursor-control technique versus a position cursor-control technique was used. Also, performance did not change when using 3,4 , or 12 surface electrodes. In the second experiment, subjects commanded a six degree-of-freedom virtual hand into seven functional postures without training, with completion rates of $82+/-4 \%$, movement times of $3.5+/-0.2 \mathrm{~s}$, and path efficiencies of $45+/$ $-3 \%$. Subjects retained the ability to use the postural controller at a high level across days after a single $1 \mathrm{~h}$ training session. Our results substantiate the novel algorithm for a postural controller as a robust and advantageous design for a MEC of multifunction prosthetic hands.
\end{abstract}

Key words: biomechatronics, electromyography, EMG, hand, limb prosthesis, multifunctional prosthetic hand, myoelectric control, postural control, transradial prosthesis, upper-limb prosthesis.

\section{INTRODUCTION}

The human hand is a complex and robust system capable of powerful grasps and fine manipulation. The musculoskeletal system in the hand consists of at least 18 joint articulations controlled by over 30 muscles [1]. Replacement of this system demands solutions to intensive engineering challenges, including the development of high bandwidth neural interfaces, intelligent control systems, and robust electromechanical systems to recreate what was lost.

Abbreviations: $\mathrm{CR}=$ completion rate, $\mathrm{D} 1-3=$ Days $1-3$, DoA $=$ degree-of-actuation, $\mathrm{DoF}=$ degree-of-freedom, $\mathrm{EMG}=$ electromyography, IMES = implantable myoelectric sensors, JAT = joint angle transform, $\mathrm{MEC}=$ myoelectric control system, $\mathrm{MT}=$ movement time, $\mathrm{nTar}=$ no target, $\mathrm{PC}=$ postural control, $\mathrm{PCA}=$ principal component analysis, $\mathrm{PE}=$ path efficiency, $\mathrm{PP}=$ palmer prehension, $\mathrm{Pt}=$ pretest, $\mathrm{RMS}=$ root mean square, $\mathrm{S} 1-$ $4=$ subjects $1-4, T=$ test, Tar $=$ target, $\mathrm{Tp}=$ target posture, $\mathrm{TMR}=$ targeted muscle reinnervation, $\mathrm{VH}=$ virtual hand, $\mathrm{VSA}=$ vector summation algorithm.

*Address all correspondence to Jacob L. Segil, PhD; Department of Mechanical Engineering, University of Colorado at Boulder, 522 UCB, Boulder, CO 80309-0427; 303735-7313; fax: 303-492-3498.

Email: jacob.segil@colorado.edu

http://dx.doi.org/10.1682/JRRD.2014.05.0134 
Multifunctional myoelectric prosthetic hands ${ }^{*}$ have been developed for several decades and recently have become commercially available [2]. These devices contain many degrees-of-actuation (DoAs) (i.e., number actuators) and can produce multiple grasping postures using myoelectric control systems (MECs). MECs attempt to decipher user intent in real time and provide an intuitive control interface (i.e., the lost function is recreated using physiologically appropriate neural commands) by measuring electromyography (EMG) signals. However, the MECs currently used restrict these multifunctional devices to a single function (i.e., open/close). This is due to the challenge of deciphering user intent from surface EMG. The further development of effective MECs will substantially progress the field of upper-limb prosthetic control.

The first MECs implemented a direct control scheme [3-5]. Direct control schemes map a single EMG control signal (i.e., control site) to a single control variable, such as joint position or motor speed. Direct control schemes require little cognitive effort from the user (if physiologically appropriate control sites are used), can occur with minimal computational delay, and require a minimal number of EMG control sites [6]. Many commercial devices on the market today successfully implement direct control schemes, such as the Motion Control Electric Terminal Device (Motion Control, Inc; Salt Lake City, Utah), Hosmer Terminal Device (Hosmer, Inc; Campbell, California), and Ottobock System Electric Hand (Ottobock Healthcare, Inc; Austin, Texas). However, these devices have a single DoA; they are "openclose" prostheses because of the MEC and mechanical design. Users indicate that multiple grasps and increased articulation are highly desirable design considerations [7-8], but these functions are not possible when using single DoA prostheses with a direct MEC.

In order to take advantage of the intuitive nature of direct MEC schemes, several techniques have been developed to increase the number of physiologically appropriate control signals available to the MEC, including targeted muscle reinnervation (TMR) [9] and implantable myoelectric sensors (IMES) [10]. TMR has shown great success for high-level upper-limb amputees [9]. Studies have successfully simulated IMES in human

\footnotetext{
*Other literature refers to these devices as multigrasp, multiple degree-of-freedom, and/or advanced prosthetic hands.
}

subjects using fine-wire intramuscular electrodes [11], and the technique has recently been implemented in humans for the first time [12].

More recent MEC techniques for multifunctional prosthetic hands include pattern recognition systems, state machines, and postural control (PC) schemes. Pattern recognition is a widely researched topic for the control of multifunctional prosthetic limbs [13-15]. It is based on measuring patterns of surface EMG signals and assigning each pattern to a desired posture or motion. Pattern recognition provides an intuitive method for controlling a prosthesis with multiple degrees-of-freedom (DoFs) (i.e., number of input signals) but lacks clinical robustness [16-18].

State machines, also referred to as event-driven finite-state schemes or binary control schemes, consist of many predefined states each with a unique function (i.e., posture or motion) that can be selected sequentially using a trigger command [19-22]. The trigger command is a physiologically inappropriate method to indicate intent; however, state machines are proven to be a clinically robust interface [23]. In fact, several commercially available prosthetic hands utilize simple state machines today. ${ }^{\dagger}$

$\mathrm{PC}$ is a technique that uses surface EMG signals to drive a cursor in a continuous two-dimensional domain, which is then transformed into a hand posture. In practice, the user is blind to the cursor in the two-dimensional domain and simply modulates EMG activity to morph the hand posture into various functional grasps. PC could be considered a type of direct control except that it includes a dimensionality augmentation step caused by a linear transform. The linear transform (hereafter referred to as a joint angle transform [JAT]) is composed of two postural vectors. The postural vectors define a two-dimensional domain, namely the PC domain, which contains various functional grasps. Several studies in both the robotic and prosthetic control literature used principal component analysis (PCA) to derive the postural vectors [24-26]. These studies were based on work by Santello et al. that found that 80 percent of the variance in grasping everyday objects can be explained using the first two principal components [27]. In other words, the linear combination

${ }^{\dagger}$ iLIMB. Touch Bionics; Mansfield (MA). http://www.touchbionics.com/ products/active-prostheses/i-limb-ultra and bebionic v3. Steeper USA; San Antonio (TX). http://bebionic.com/the hand 
of two postural vectors could accurately reproduce the hand postures needed for grasping everyday objects.

Other studies project EMG signals to a two-dimensional domain similar to the PC domain. De Rugy et al. used forearm EMG signals for a two-dimensional target-acquisition task in order to study muscle coordination under various biomechanical conditions [28]. Pistohl et al. controlled individual digits of a virtual hand $(\mathrm{VH})$ and prosthetic hand using intrinsic hand EMG by maneuvering a cursor in a two-dimensional domain, allowing for simultaneous and proportional control of multiple DoFs [29]. Radhakrishnan et al. studied users' ability to learn novel myoelectric control interfaces using a two-dimensional center-out target-acquisition task [30]. However, none of these studies focused on the clinical implementationproducing functional postures and using clinically available surface EMG control sites - as we did here using the novel PC algorithm.

Previously, we found that a specific mapping of the EMG signals in the PC domain augmented the ability of subjects to drive a $\mathrm{VH}$ into functional postures when using the principal components derived by Santello et al. [31]. This work builds upon that finding by developing a novel algorithm for a postural controller that is not dependent on PCA to derive the postural vectors. Namely, the postural vectors that compose the JAT are defined dynamically as the user navigates the PC domain and are dependent on the two nearest postures within the PC domain map (a spatially and temporally dependent algorithm).

\section{POSTURAL CONTROL ALGORITHM}

The novel algorithm of the postural controller is detailed in Figure 1. In general, the controller transforms an array of EMG signals into a joint angle array (i.e., a hand posture) using a temporally and spatially dependent linear transform. In practice, the user modulates EMG activity (such as using the wrist as a joystick) to morph the hand posture of the prosthesis without the need for trigger signals to change state. Many parameters within this algorithm can be adjusted in order to build the most effective and intuitive interface and are discussed in more detail below. The experiments discussed here compared several design parameters and quantified the clinical efficacy of the controller.

\section{Electromyography Acquisition}

An untargeted surface electrode array on the dominant forearm (Figure 2(a)) acquires myoelectric signals. The array spans the circumference of the proximal third of the forearm with $N$ electrodes (depending on the experiment) in the longitudinal direction. The humeral epicondyles locate the $y$-axis and therefore orientate the array with respect to the arm. The untargeted array designates that specific muscles are not targeted by each electrode. Standard clinical techniques process raw EMG signals into an array of root mean square (RMS) averages over $100 \mathrm{~ms}$ nonoverlapping time windows. The EMG acquisition results in a "snapshot" of EMG activity

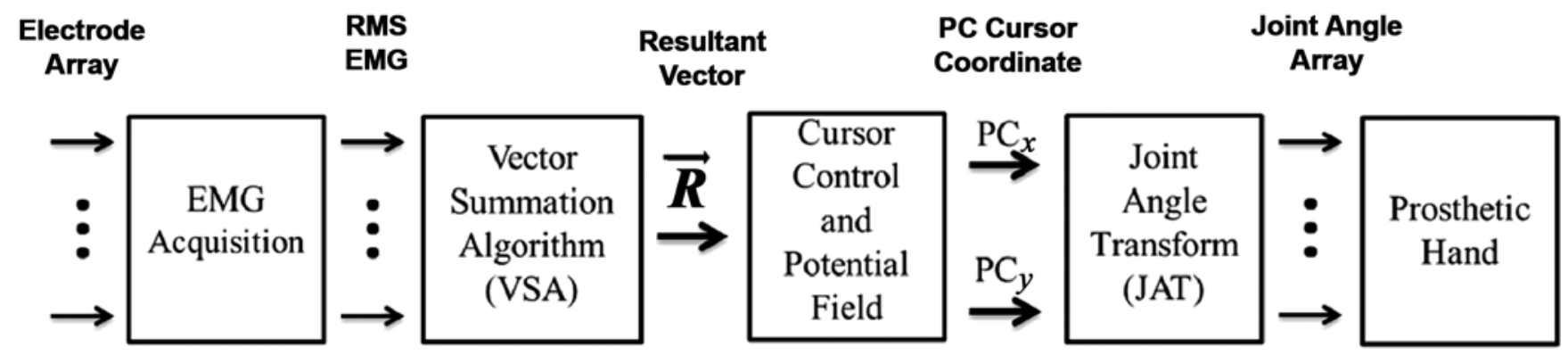

Figure 1.

Novel algorithm for postural controller. Untargeted surface electrode array acquires electromyography (EMG) signals. Filtered root mean square (RMS) average EMG values are passed to vector summation algorithm (VSA), which produces resultant vector ( $\vec{R})$. Resultant vector is used to calculate postural control (PC) cursor coordinate $\left(\mathrm{PC}_{x}, \mathrm{PC}_{y}\right)$ using various cursor-control schemes and potential field designs. Finally, joint angle transform (JAT) transforms PC cursor coordinate to joint angle array, which is sent to prosthetic hand. 
(a)

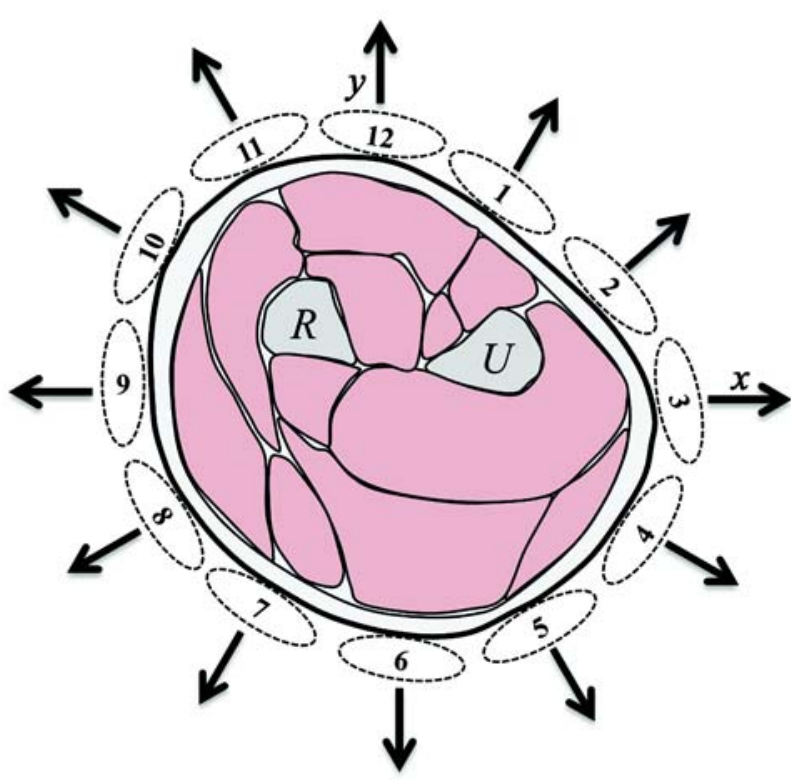

(c)

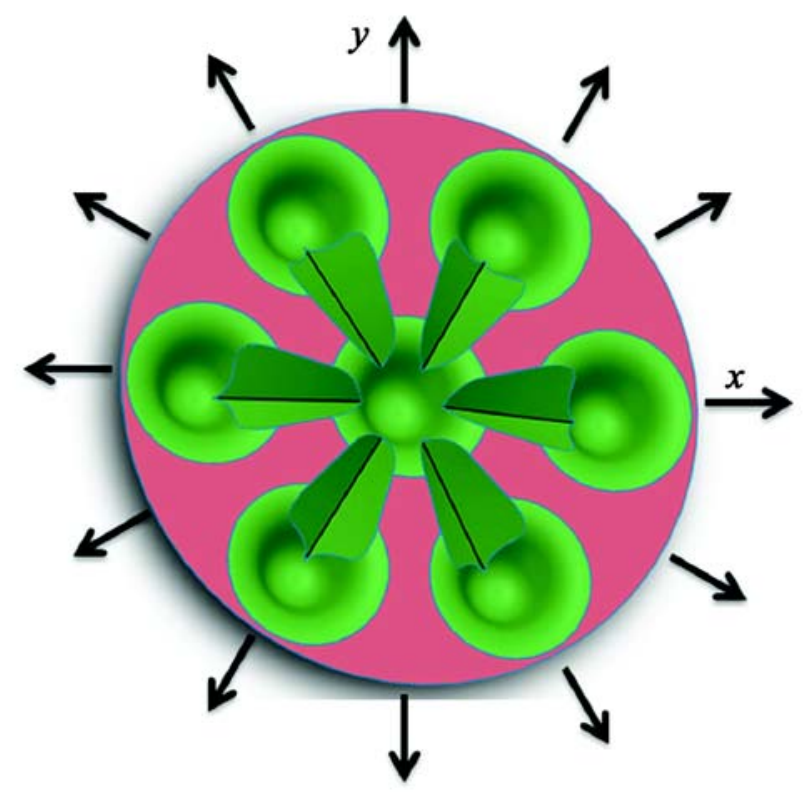

(b)

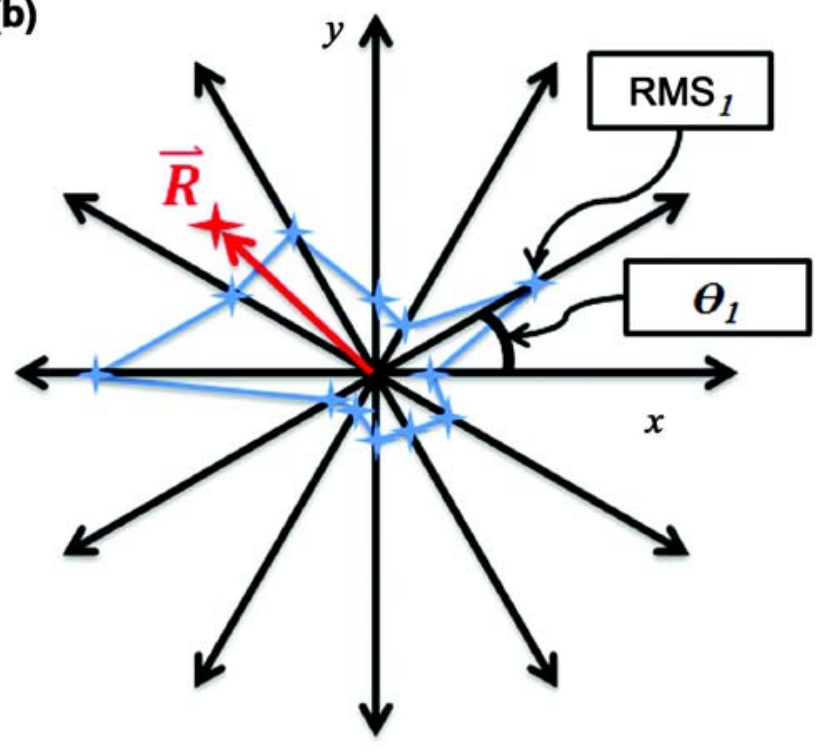

(d)

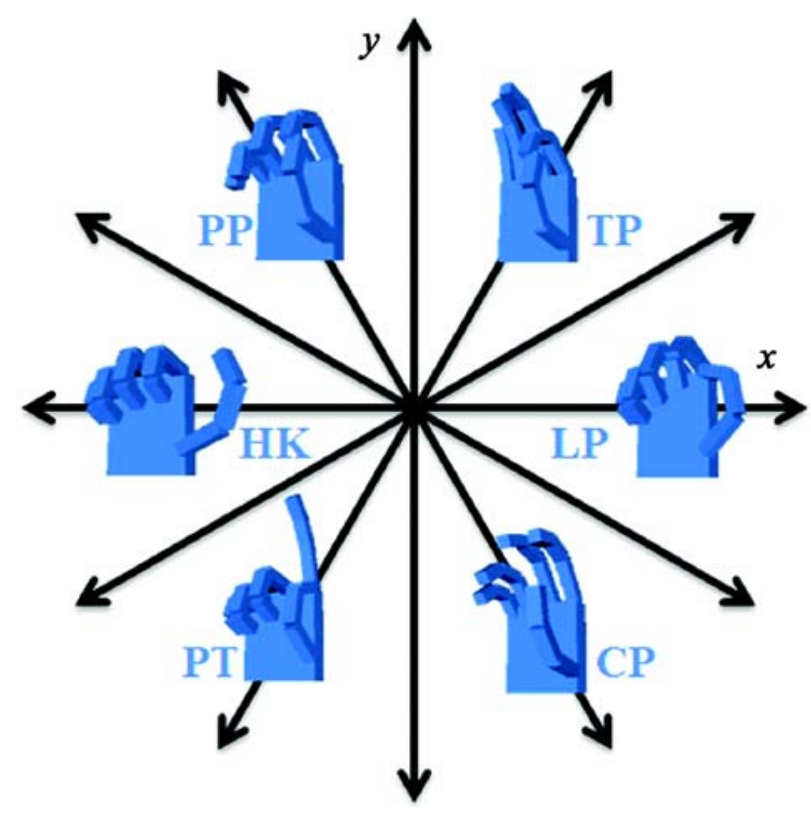

Figure 2.

Components of postural controller. (a) Untargeted electrode array (1-12) is arranged about cross section of forearm. $R=$ radius bone, $U=$ ulnar bone. (b) Vector summation map depicts root mean square (RMS) electromyography activity as measured by electrode array. Vector summation algorithm calculates resultant vector $(\vec{R}) \cdot \theta=$ joint angle array. (c) Example potential field design where red/green areas distinguish areas of zero/negative potential, respectively. This potential field design was used in Experiment B. (d) Exemplary postural map with seven postures arranged in symmetric distribution about postural control domain (hand flat posture not shown at origin). This postural map design was used in Experiment B. CP = cylindrical prehension, $\mathrm{HK}=$ hook, $\mathrm{LP}=$ lateral prehension, $\mathrm{PP}=$ palmar prehension, $\mathrm{PT}=$ pointer, $\mathrm{TP}=$ tip prehension . 
within the forearm, which the vector summation algorithm (VSA) deciphers further.

\section{Vector Summation Algorithm}

The VSA interprets the snapshot of EMG activity using a uniformly spaced vector summation map (Figure 2(b)). Electrodes on the dorsal/ventral side of the limb (wrist extension/flexion) correspond to the $y$-axis of the PC domain, and electrodes on the medial/lateral side of the limb (ulnar/radial deviation) correspond to the $x$-axis of the PC domain (for a right-sided limb). The RMS EMG value determines the magnitude of each corresponding vector (Figure 2(b)). The summation of all vectors produces a resultant vector $(\vec{R})$. Equation 1 describes the calculation of $\vec{R}$ :

$$
\vec{R}(t)=\left[\begin{array}{l}
R_{x}(t) \\
R_{y}(t)
\end{array}\right]=\sum_{i=1}^{N}\left[\begin{array}{l}
\operatorname{RMS}_{i}(t) \cos \theta_{i} \\
\operatorname{RMS}_{i}(t) \sin \theta_{i}
\end{array}\right],
$$

where $\mathrm{RMS}_{i}$ are the RMS values of the $\mathrm{EMG}_{i}$ signal, $\theta_{i}$ is the control site angle with respect to the $x$-axis, $N$ is the number of control sites, and $t$ is time. The direction of the resultant vector indicates the area in the forearm with the most EMG activity, and the magnitude indicates the relative amount of EMG activity. In short, the VSA reduces the RMS EMG array into a single resultant vector $(\vec{R})$ that subsequently drives the $\mathrm{PC}$ cursor coordinate $\left(\mathrm{PC}_{x}, \mathrm{PC}_{y}\right)$.

\section{Cursor-Control Schemes and Potential Field}

The resultant vector produced by the VSA $(\vec{R})$ controls the $\mathrm{PC}$ cursor coordinate $\left(\mathrm{PC}_{x}, \mathrm{PC}_{y}\right)$ using a position or velocity cursor-control scheme (compared in Experiment A). These methods were compared since they affect the way the prosthesis functions for the user. A position control scheme equates EMG activity with joint angles, whereas a velocity control scheme equates EMG activity with motor speed and direction. For a user, a short muscle contraction would cause the hand to move to a position and then return to hand flat posture when using a position control scheme; in contrast, the same muscle contraction would cause the hand to move at a certain speed and then stop when using the velocity control scheme. The position control scheme interprets the resultant vector as a positional command vector (i.e., units of distance). In the position cursor-control scheme, the endpoint of the resultant vector equals the PC cursor coordinate (Equation 2):

$$
\left[\begin{array}{l}
\mathrm{PC}_{x}(t) \\
\mathrm{PC}_{y}(t)
\end{array}\right]=\left[\begin{array}{l}
R_{x}(t) \\
R_{y}(t)
\end{array}\right]=\vec{R}(t) \quad .
$$

The velocity cursor-control scheme interprets the resultant vector as a velocity command vector (i.e., units of distance/time). A discrete integration over time determines the cursor position (Equation 3):

$$
\left[\begin{array}{l}
\mathrm{PC}_{x}(t) \\
\mathrm{PC}_{y}(t)
\end{array}\right]=V_{\text {gain }} * \sum_{j=1}^{J}\left(\vec{R}_{j}(t) * \Delta t_{j}\right),
$$

where $(\vec{R})$ is the instantaneous resultant vector, $\Delta t_{j}$ is the loop time, $V_{\text {gain }}$ is the velocity gain, and $j$ is the software loop count (up to the total loop count, J). The velocity gain adjusts the maximum allowable speed (i.e., a speed limit). In practice, the magnitude of the resultant vector corresponds to the speed of the cursor and the direction of the resultant vector corresponds to the direction the cursor moves.

The potential field (Figure 2(c)) was developed in order to augment the ability of the user to perform functional grasps using the postural controller. The potential field preferentially attracts the cursor coordinate to certain regions in the PC domain using a position feedback loop with a proportional-derivative controller. The practical effect is that the hand seems to snap into the preferred functional postures. This occurs because the position feedback loop acts like a gravitational (potential) field that pulls the cursor toward certain positions in the PC domain, similar to a ball rolling down a ramp. The feedback controller forces the cursor to the areas of lowest potential (i.e., the bottom of the wells and wedges) but still allows for intermediate positions between wells when EMG activity is produced that opposes the potential field. For example, the instantaneous cursor position depicted by the star in Figure 2(b) is on the edge of the potential well for the palmer prehension (PP) grasp (Figure 2(d)). The cursor position will move toward the PP target posture (Tp) in the subsequent time steps due to the potential well (unless the EMG activity changes drastically). All parameters of the potential field (diameter of the wells, width of the wedges, and depth of wells and wedges) can be adjusted in order to best aid the user in performing functional grasps. In effect, the potential field can be visualized as a third dimension to the PC domain as illustrated in Figure 2(c), where the red/green areas 
have zero/negative potential, respectively. The design of the potential fields was determined during pilot studies in which various geometries (i.e., only wells, only wedges, and both wells/wedges) were compared. The parameters found during the pilot study were held constant for all subjects throughout the experiments described here. The sequential processing of the resultant vector by the cursor-control scheme and then the potential field produces a PC cursor coordinate $\left(\mathrm{PC}_{x}, \mathrm{PC}_{y}\right)$ that the JAT converts into a hand posture.

\section{Joint Angle Transform}

The JAT converts the PC cursor coordinate into a hand posture based on the postural map (Figure 2(d)) at a rate of $10 \mathrm{~Hz}$. The postural map defines the number and location of the grasps (targets) available to the user in the PC domain. Table 1 defines the joint angles of each Tp (including hand flat). Each cursor coordinate in the PC domain corresponds to a unique posture. When the PC cursor coordinate equals the target position, then the controller reproduces the Tp identically. As the PC cursor coordinate moves between Tps, the controller produces a linear combination of those two postures. A representative postural map is shown in Figure 2(d) (the hand flat posture is located at the origin of the PC domain but is not shown). This new algorithm allows for a vast number of postures and complete freedom to position the postures within the PC domain.

The JAT is a temporally and spatially dependent linear transform that converts the PC cursor coordinate into a joint angle array. The generalized equation (Equation 4) defines the JAT: $\boldsymbol{\theta}=\left[\begin{array}{c}\theta_{1} \\ \theta_{2} \\ \theta_{3} \\ \theta_{4} \\ \theta_{5} \\ \theta_{6}\end{array}\right]=\mathbf{J A T} *\left[\begin{array}{lll}\mathrm{PC}_{x}(t) \\ \mathrm{PC}_{y}(t)\end{array}\right]=\left[\begin{array}{lll}\mathrm{JAT}_{1,1}\left(\mathrm{PC}_{x}, \mathrm{PC}_{y}\right) & \mathrm{JAT}_{1,2}\left(\mathrm{PC}_{x}, \mathrm{PC}_{y}\right) \\ \mathrm{JAT}_{2,1}\left(\mathrm{PC}_{x}, \mathrm{PC}_{y}\right) & \mathrm{JAT}_{2,2}\left(\mathrm{PC}_{x}, \mathrm{PC}_{y}\right) \\ \mathrm{JAT}_{3,1}\left(\mathrm{PC}_{x}, \mathrm{PC}_{y}\right) & \mathrm{JAT}_{3,2}\left(\mathrm{PC}_{x}, \mathrm{PC}_{y}\right) \\ \mathrm{JAT}_{4,1}\left(\mathrm{PC}_{x}, \mathrm{PC}_{y}\right) & \mathrm{JAT}_{4,2}\left(\mathrm{PC}_{x}, \mathrm{PC}_{y}\right) \\ \mathrm{JAT}_{5,1}\left(\mathrm{PC}_{x}, \mathrm{PC}_{y}\right) & \mathrm{JAT}_{5,2}\left(\mathrm{PC}_{x}, \mathrm{PC}_{y}\right) \\ \mathrm{JAT}_{6,1}\left(\mathrm{PC}_{x}, \mathrm{PC}_{y}\right) & \mathrm{JAT}_{6,2}\left(\mathrm{PC}_{x}, \mathrm{PC}_{y}\right)\end{array}\right]\left[\begin{array}{l}\mathrm{PC}_{x}(t) \\ \mathrm{PC}_{y}(t)\end{array}\right]$

where $\mathrm{PC}_{x}(t)$ and $\mathrm{PC}_{y}(t)$ are the temporally dependent $\mathrm{PC}$ cursor coordinates based on the acquired EMG signals, $\mathrm{JAT}_{k, l}$ are the joint angles for the two closest postures to the current PC cursor coordinate as determined by the postural map ( $k=1-6$ for a six DoF hand, $l=1-2$ corresponding to the two closest postures), and $\theta$ is the joint angle array. At any moment in time, the columns of the JAT are made up of two columns of Table 1 depending on the two nearest Tps. A novel aspect of this PC scheme is that the JAT is spatially dependent (i.e., the columns of the JAT change depending on the PC cursor coordinate). This fact differentiates this work from previous myoelectric control algorithms. Also, the spatial dependence of the JAT allows for the freedom to position postures within the PC domain without limitation. This algorithm allows for any number of Tps that can be placed in any arrangement within the PC domain. The JAT ensures that the hand posture morphs as the cursor moves between targets in the PC domain.

This novel algorithm for a postural controller includes many customizable features (number of electrodes, cursor-control schemes, potential field designs, postural map designs, etc.). This fact motivated Experiment $\mathrm{A}$, in which the preferred number of electrodes and cursor-control scheme was determined. Afterward, Experiment $\mathrm{B}$ was designed to test the clinical efficacy of the postural controller by quantifying the controller performance in a simulated real-world setting. Experiment B also studied the effects of visual feedback on performance and the learning rate across days.

Table 1.

Joint angles for 6 degree-of-freedom prosthetic hand for seven functional grasps.

\begin{tabular}{|c|c|c|c|c|c|c|c|}
\hline \multirow{2}{*}{ Joint Angle $\left(^{\circ}\right)$} & \multicolumn{7}{|c|}{ Grasp } \\
\hline & $\mathbf{L P}$ & $\mathbf{T P}$ & $\overline{\mathbf{P P}}$ & HK & $\mathbf{P T}$ & $\mathbf{C P}$ & HF \\
\hline Thumb Rotation & 20 & 90 & 90 & 0 & 0 & 90 & 0 \\
\hline Thumb Flexion & 90 & 65 & 65 & 90 & 90 & 65 & 0 \\
\hline Index Flexion & 70 & 70 & 70 & 70 & 0 & 45 & 0 \\
\hline Middle Flexion & 80 & 0 & 80 & 80 & 80 & 55 & 0 \\
\hline Ring Flexion & 80 & 0 & 80 & 80 & 80 & 65 & 0 \\
\hline
\end{tabular}




\section{METHODS}

Eleven total subjects naive to myoelectric control completed an experiment using the described postural controller. Experiment A consisted of a target-acquisition task using various configurations of the controller and was completed by seven subjects in order to empirically derive the best cursor-control scheme and electrode configuration. Experiment B consisted of a posture-matching exercise using various forms of visual feedback over $3 \mathrm{~d}$ and was completed by four subjects (S1-S4) in order to measure the ability of the users when performing a more clinically oriented task. All subjects were nondisabled and claimed to have normal vision and upper-limb function. They conducted the experiment using the dominant limb (10 right-hand dominant subjects). An experimental meeting took approximately $2 \mathrm{~h}$. The Institutional Review Board at the University of Colorado at Boulder reviewed and approved all experimental protocols. Informed consent was obtained from all subjects before each experiment.

\section{Apparatus}

Subjects sat in an upright position, with their dominant arm bent at the elbow and forearm pronated on the armrest of the chair in front of a computer monitor. Experiments were carried out using an untargeted electrode array as described previously (Figure 2(a)). Selfadhesive snap electrode pairs with $2 \mathrm{~cm}$ spacing (Noraxon \#272, Noraxon USA, Inc; Scottsdale, Arizona) comprised the electrode array. The array spanned the circumference of the proximal third of the forearm, with the electrodes in the longitudinal direction. A Noraxon Telemyo DTS system acquired the signals with a hardware sampling rate of $1.5 \mathrm{kHz}$. A NI-DAQ 9205 (National Instruments Corp; Austin, Texas) interfaced with the ana$\log$ output module of the Noraxon system and was controlled using a custom-built LabVIEW interface (National Instruments Corp). The LabVIEW interface processed the EMG signals using standard processing techniques (band pass $30-450 \mathrm{~Hz}$, rectification, RMS average). Individual gains, thresholds, and offsets tuned the system to produce a symmetric and comfortable system (i.e., equivalent effort caused equivalent RMS average for all sites).

\section{Experiment A}

Experiment A took place in a single experimental meeting. During a single trial, subjects performed a center-out target-acquisition task using one of two cursor- control schemes and one of three electrode configurations for a total of six control methods: 12-site position control (P12), 12-site velocity control (V12), 4-site position control (P4), 4-site velocity control (V4), 3-site position control (P3), and 3-site velocity control (V3). These experimental conditions were tested in order to empirically derive the best cursor-control scheme and electrode configuration. The sequence of control methods was presented in a pseudorandom fashion, where the velocity and position control sessions and the order of electrode configurations within each session was randomized for each subject (an example protocol is shown in Figure 3). The 4-site sessions used electrode numbers 3, 6, 9, and 12 , and the 3 -site sessions used electrode numbers 3,7 , and 11 as described in Figure 2(a). The visual feedback paradigm for all sessions consisted of the PC domain including the target circle and PC cursor coordinate. Twelve equally spaced targets with radii of 14 percent of the PC domain were set at a radius 70 percent between the origin and the edge of the PC domain and aligned with the vector summation map as described in Radhakrishnan et al.'s work [30]. Three randomized blocks of twelve targets were presented for each session. No potential field was applied during Experiment $\mathrm{A}$ in order to isolate the differences between each control method. A trial consisted of directing the cursor from the origin to the target within $10 \mathrm{~s}$ (including the $1 \mathrm{~s}$ hold time), otherwise the trial was considered a failure. Each session consisted of 36 trials and tested a single control method.

\section{Experiment B}

Experiment B took place across three experimental meetings on three separate days (D1-D3). This experiment was designed to measure the clinical efficacy of the controller, evaluate the effects of visual feedback on performance, and study learning across multiple days. In a single trial, subjects were asked to command a six DoF $\mathrm{VH}$ into one of seven Tps using a 3-site electrode array and velocity cursor-control scheme. This virtual posturematching exercise was based on the Target Achievement Control Test [32], with the addition of various visual feedback methods. Each experimental meeting was composed of the same sequence of four sessions, which presented various visual feedback paradigms: (1) Pretest (Pt) session, (2) target (Tar) training session, (3) no target (nTar) training session, and (4) test (T) session. The sequence of these sessions was meant to help train the subjects in achieving the various Tps in the PC domain. During all sessions, a computer monitor presented a Tp and a $\mathrm{VH}$ prosthesis that responded to the real-time output 


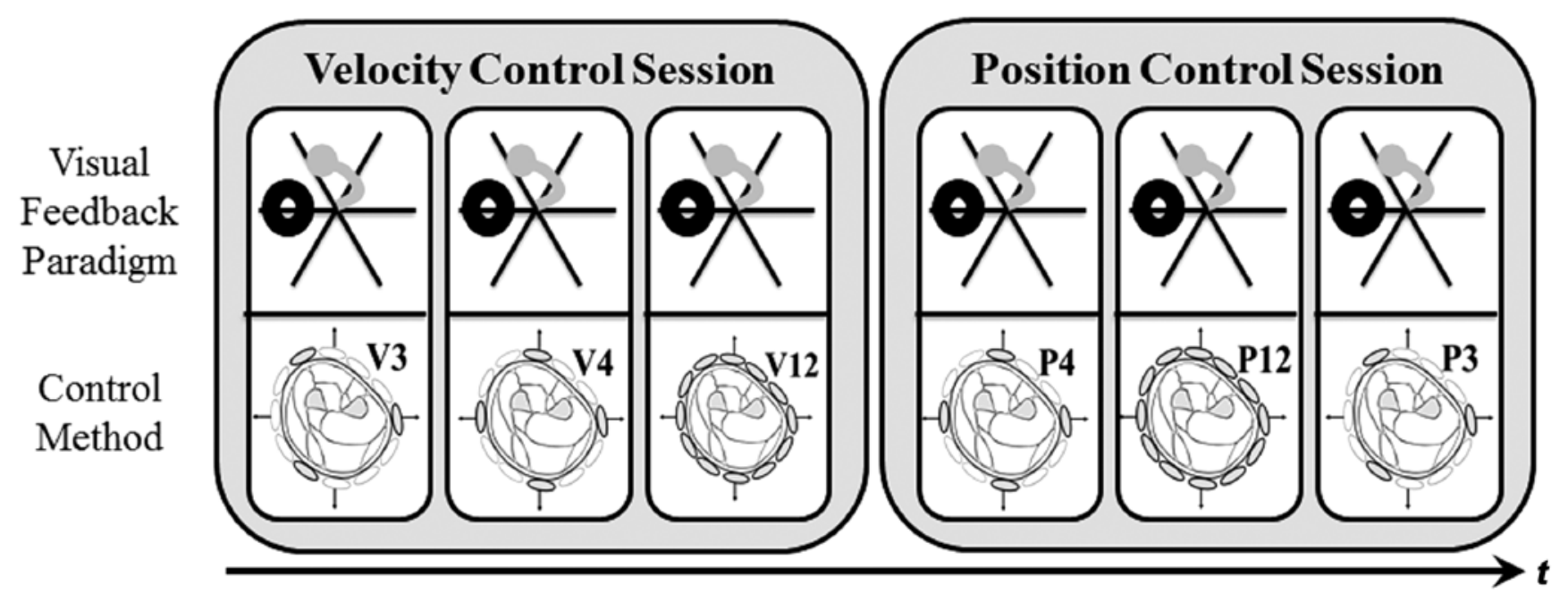

Figure 3.

Experiment A protocol for exemplary single meeting. Visual feedback paradigm for all sessions consisted of postural control (PC) domain including target circle and PC cursor coordinate. Sequence of control methods was presented in pseudorandom fashion, where velocity and position control sessions and order of electrode configurations within each session were randomized for each subject. P3 = 3-site position, P4 = 4-site position, P12 = 12-site position, V3 = 3-site velocity, V4 = 4-site velocity, V12 = 12-site velocity.

of the postural controller. The additional visual feedback differed between experimental sessions (Figure 4). The PC domain with the real-time cursor position as well as the Tp was presented in the Tar session. The PC domain with the real-time cursor position but not the $\mathrm{Tp}$ was presented in the nTar session. The PC domain was hidden during the testing sessions (Pt and $\mathrm{T}$ ). The sequence of the sessions (Pt, Tar, nTar, T) was designed to allow for the assessment of learning [33]. The $\mathrm{Pt}$ and $\mathrm{T}$ sessions simulated a real-world environment in which a screen displaying the PC domain would not be present, thereby assessing the clinical efficacy of the system. The Tar and nTar sessions used visual feedback to help train the subjects to perform the task. Six equally spaced targets with radii of 14 percent of the PC domain were located at a radius 70 percent between the origin and the edge of the PC domain (the seventh Tp, hand flat, was located at the origin of the PC domain, Figure 2(d)). A trial consisted of matching the $\mathrm{VH}$ to the Tp within $10 \mathrm{~s}$ (including the $1 \mathrm{~s}$ hold time), otherwise the trial was considered a failure. The VH matched the Tp when the cursor was within the 14 percent radii of the target in the PC domain and was provided using a visual indicator. The $\mathrm{Pt} / \mathrm{T}$ sessions consisted of 35 trials ( 5 attempts at each target), and the Tar/ nTar sessions consisted of 70 trials (10 attempts at each target). The identical potential field was applied during all experimental meetings and is depicted in Figure 2(c). The EMG tuning parameters (gains, offsets, and thresholds) were defined during the first meeting and not altered during the second or third meetings.

\section{Performance Metrics}

Several performance metrics were used to study the performance during experiments $\mathrm{A}$ and $\mathrm{B}$. The completion rate $(\mathrm{CR})$ is the number of successful trials per total number of trials. The movement time (MT) is the duration of the successful trial in seconds not including the $1 \mathrm{~s}$ hold time. The path efficiency (PE) (Equation 5) describes the degree to which the cursor trace erred from the most direct path between the origin and target during successful trials:

$$
\begin{aligned}
& \mathrm{PE}(\%)=\frac{\text { Traveled Distance }}{\text { Ideal Distance }} * 100= \\
& \frac{\sum_{j=1}^{N} \sqrt{\left(\mathrm{PC}_{x, j}-\mathrm{PC}_{x, j-1}\right)^{2}+\left(\mathrm{PC}_{y, j}-P C_{y, j-1}\right)^{2}}}{\sqrt{\left(\text { Target }_{x}\right)^{2}+\left(\text { Target }_{y}\right)^{2}}} * 100
\end{aligned}
$$

The traveled distance is the discrete integral of the PC cursor coordinate path. The ideal distance is the straight-line 


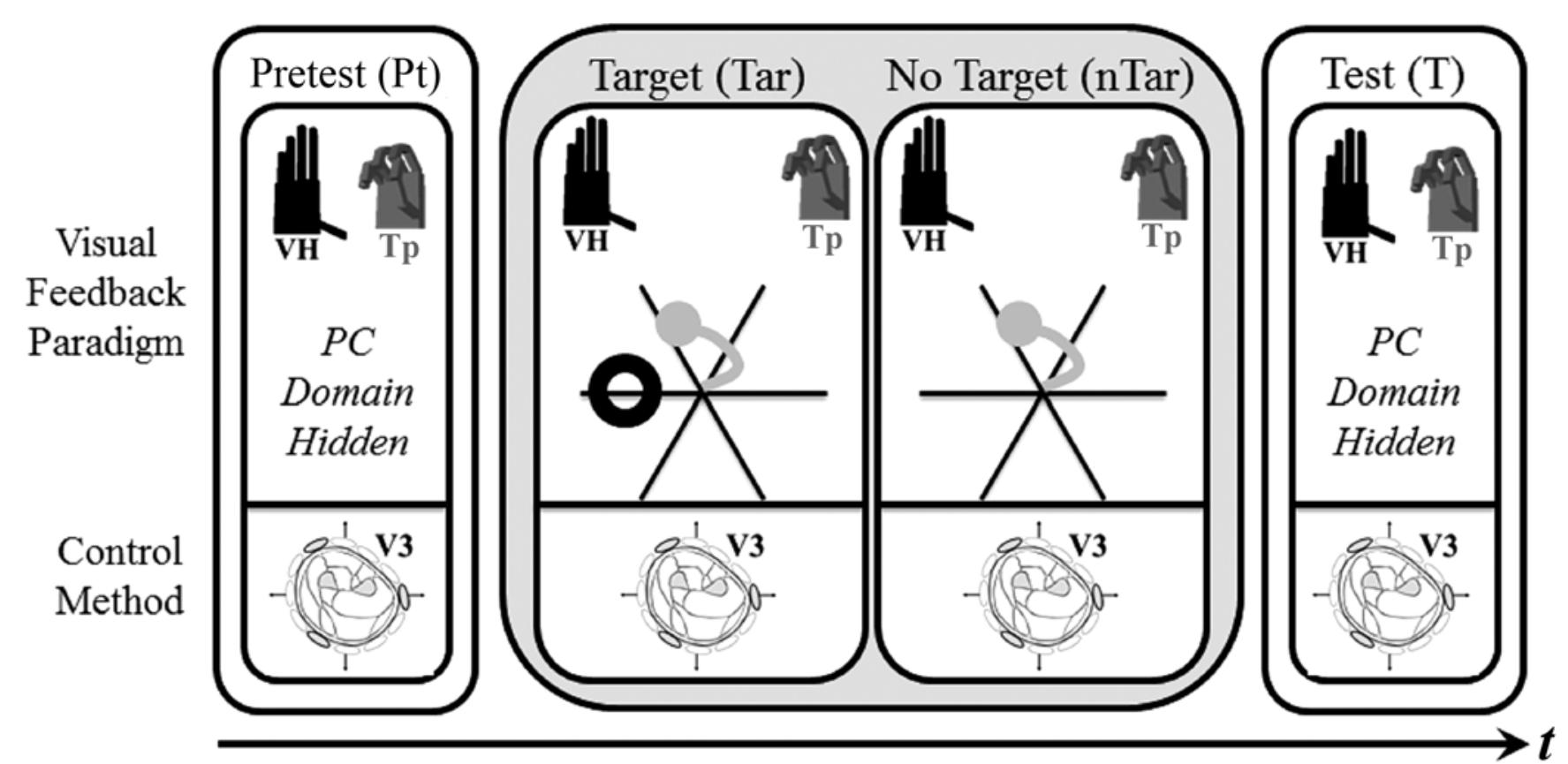

Figure 4.

Experiment B single meeting protocol. This protocol was repeated on days 1-3. During all sessions, computer monitor presented dynamic virtual hand $(\mathrm{VH})$ prosthesis that responded to real-time output of postural controller and target posture (Tp). Additional visual feedback differed between pretest (Pt)/test ( $\mathrm{T})$, target (Tar), and no target (nTar) sessions. All sessions used the same control method (V3 = 3-site velocity control). $\mathrm{PC}=$ postural control, $t=$ time.

distance between the target coordinate and the origin. A $\mathrm{PE}$ equal to 100 percent signifies that the cursor traveled along a straight line between the origin and target.

MT and PE were only reported for successful trials. Better performance is quantified by higher CR, lower MT, and higher PE. One- or two-factor analyses of variance and Tukey-Kramer post hoc analyses were used when appropriate, with a significance level of 0.05 . Experimental results report mean \pm standard error of the mean.

\section{RESULTS}

\section{Experiment A}

The PC cursor coordinate traces in the PC domain using the six control methods for a single subject are shown in Figure 5. Figure 5(a) displays the successful trials and Figure 5(b) displays the failed trials for a single subject across all six conditions. The 12 targets are shown as well as the vector summation map. The top row depicts the position control trials and the bottom row depicts the velocity control trials. Figure 5 describes qualitatively an increase in $\mathrm{CR}$ and $\mathrm{PE}$ for the velocity control sessions compared to the position control sessions, shown by the increase in straighter traces. Also, performance does not seem to change when using a 3-, 4-, or 12-site electrode array. These qualitative observations are tested statistically using the performance metrics described previously.

The average performance metrics across subjects for each experimental session are shown in Figure 6. All metrics described the same two findings: the velocity control method allowed for better control than the position control method and the number of control sites did not change the performance. In detail, the $\mathrm{CR}$ was significantly greater with velocity control $(84 \pm 3 \%)$ than with position control $(45 \pm 3 \%, p<0.001)$. The MT was significantly lower with velocity control $(5.3 \pm 0.2 \mathrm{~s})$ than with position control $(6.1 \pm 0.1 \mathrm{~s}, p<0.001)$. The PE was significantly greater with velocity control $(69 \pm 3 \%)$ than with position control $(27 \pm 2 \%, p<0.001)$. In addition, the number of control sites used during each session did not affect the CR, MT, or PE ( $p=0.57,0.34$, and 0.32 , respectively). 
(a)
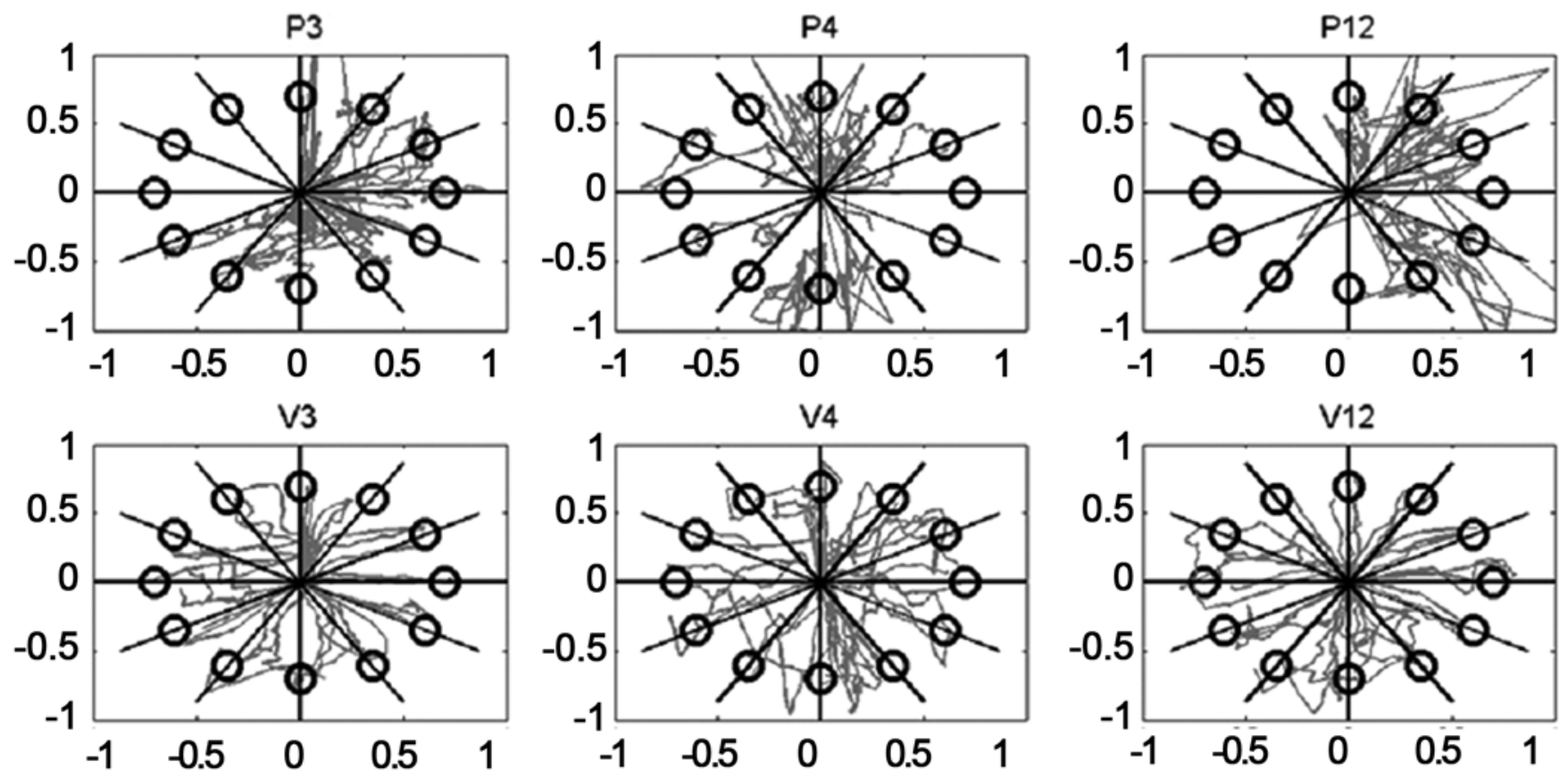

(b)
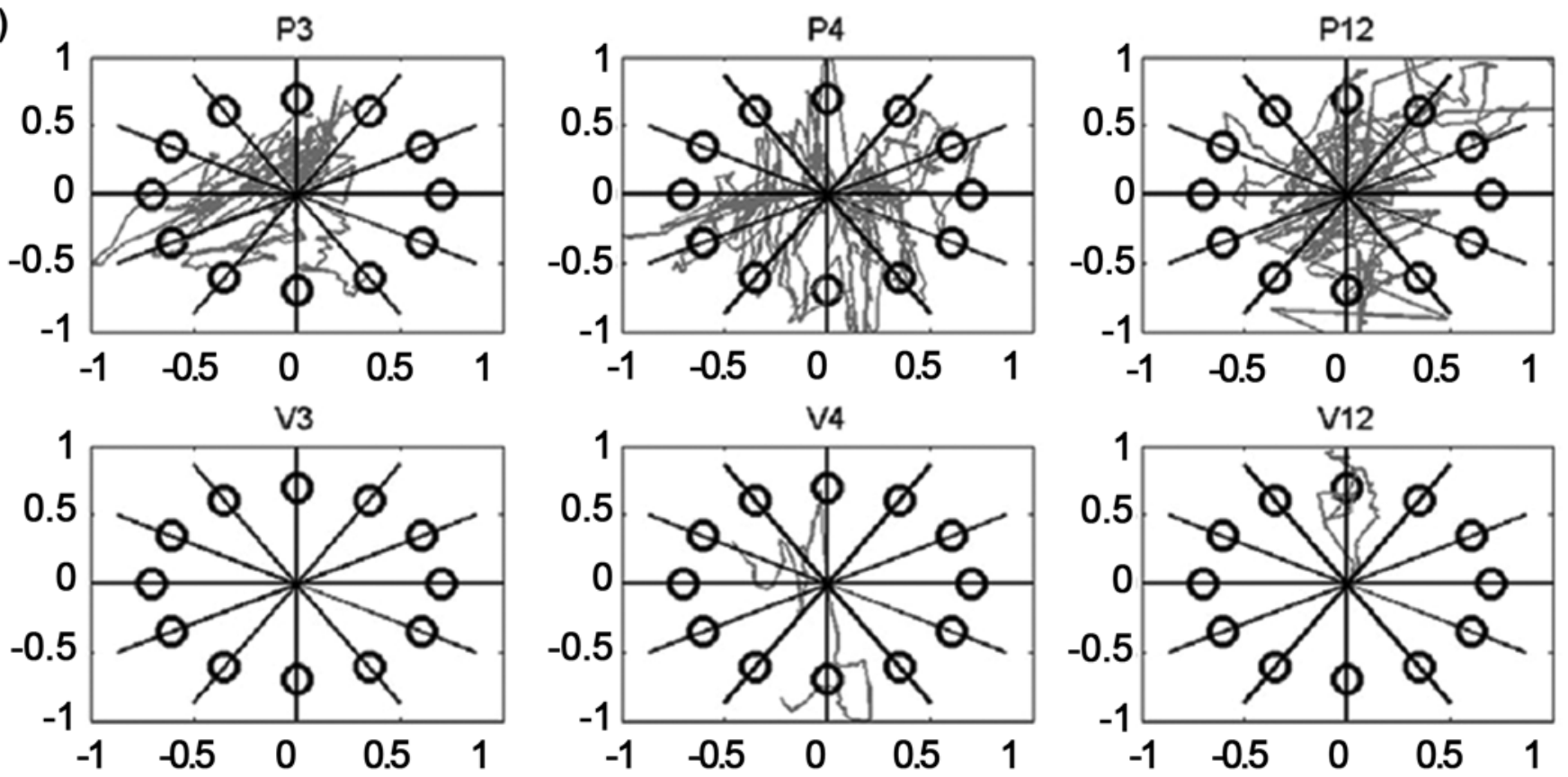

Figure 5.

Raw postural control (PC) cursor coordinate traces by single subject. (a) Successful attempts for each experimental session. (b) Failed attempts for each experimental session. Targets are shown as circles. Axes correspond to 12 electrodes on limb. P3 = 3-site position, P4 = 4-site position, P12 = 12-site position, V3 = 3-site velocity, V4 = 4-site velocity, V12 = 12-site velocity. 

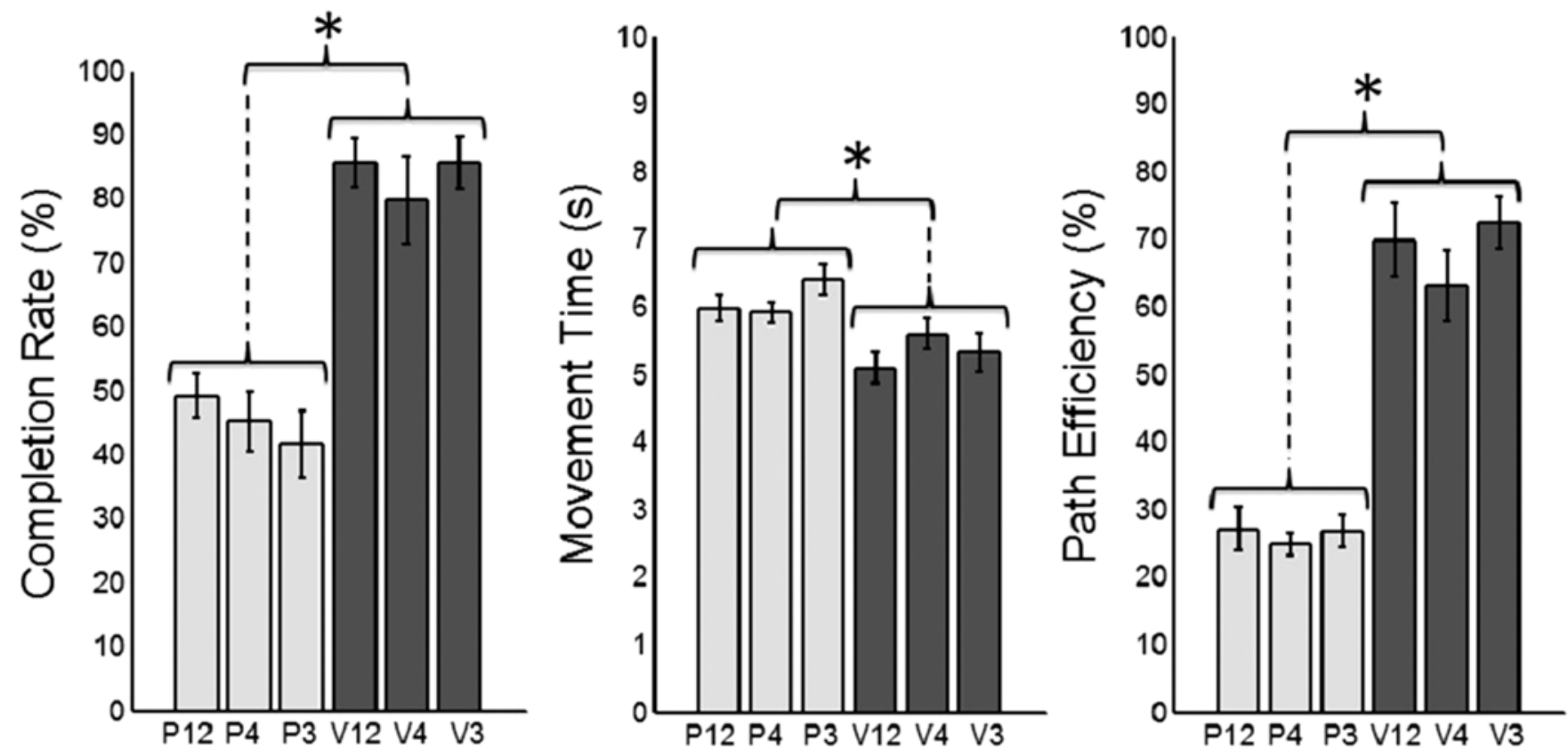

Figure 6.

Experiment A performance metrics averaged across subjects for each control method. No difference was found when comparing 12site, 4-site, and 3-site electrode array sessions. ${ }^{*} p<0.05$. P3 = 3-site position, P4 = 4-site position, P12 = 12-site position, V3 = 3site velocity, V4 = 4-site velocity, V12 = 12-site velocity.

\section{Experiment B}

The ability of subjects to volitionally command a six DoF VH into seven functional postures was quantified (Figure 7) using the average CR, MT, and PE performance metrics for each subject (S1-S4). During the testing session, the average CR, MT, and PE across subjects was $82 \pm 4$ percent, $3.5 \pm 0.2 \mathrm{~s}$, and $45 \pm 3$ percent. The CR for $\mathrm{S} 4$ is a statistical outlier $(p=0.01)$ and is discussed in more detail below. The average $\mathrm{CR}$ without $\mathrm{S} 4$ is $88 \pm$ 2 percent.

The retention of the ability to use the postural controller was tested by comparing performance metrics produced during the Pt sessions for all subjects across days (Figure 8). The Pt session occurred before the training sessions and thereby tested the retention of the ability to use the postural controller from the previous day. The CR and PE results were significantly different across days ( $p<$ 0.001 and $p=0.002$, respectively). Post hoc analysis showed that the CR and PE for D1 was significantly lower than for D2-3. The CR and PE results showed no difference between D2 and D3 $(p=0.47$ and $p=0.08$, respectively). The MT results showed no difference across days $(p=0.98)$.

The effect of visual feedback on performance was tested by comparing average metrics across days for the Tar, nTar, and T sessions (Table 2). The CR was significantly greater during Tar and nTar sessions than during $\mathrm{T}$ session for three out of four subjects. The MT and PE did not change for any subjects across visual feedback paradigms (average $p$-values of $0.50 \pm 0.05$ and $0.83 \pm 0.08$ ). These results indicate the additional visual feedback provided during Tar and nTar sessions augments $\mathrm{CR}$, but not MT or PE. In other words, the additional visual feedback increased the frequency of successful trials but did not increase the speed or precision of the successful trials.

\section{DISCUSSION}

\section{Experiment A}

The results of Experiment A described a significant increase in performance using a velocity cursor-control scheme compared to a position cursor-control scheme. 

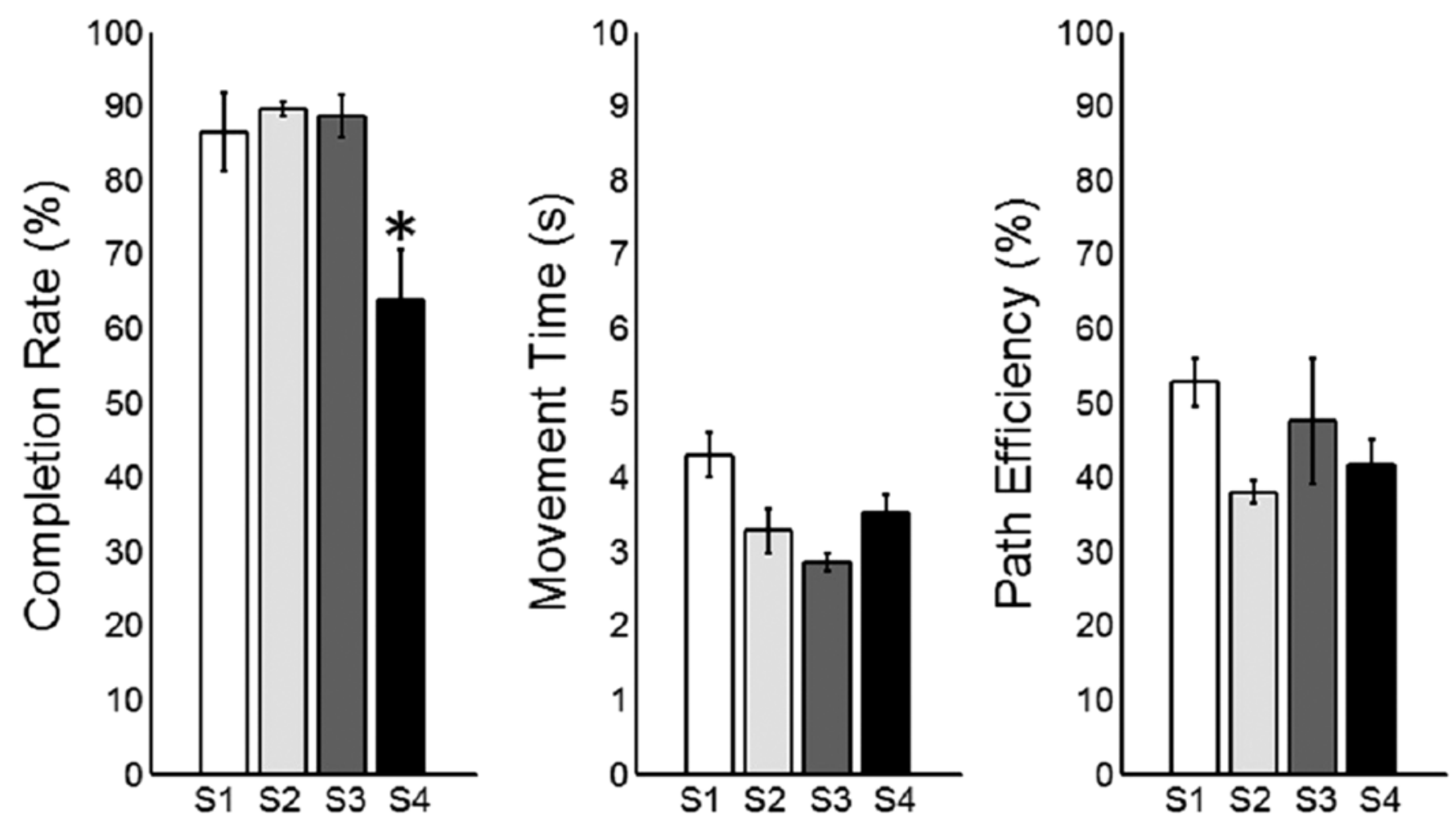

Figure 7.

Experiment B testing session performance metrics averaged over days for each subject (S). ${ }^{*} p<0.05$.

Several factors were observed that differentiate the two cursor-control schemes. The position control scheme requires continuous activation of specific musculature in order to complete a successful trial because quiescent EMG activity (i.e., rest) equates to the $\mathrm{PC}$ cursor coordinate position at the origin. It was observed that subjects had difficulty holding specific muscular contractions in order to maintain the cursor in the target position. The velocity control scheme allowed for sequential activations of specific musculature in order to complete a successful trial because quiescent EMG activity equates to a stationary PC cursor coordinate position. As a result, subjects were observed using a "pulsing" strategy, in which sequential muscle contractions moved the cursor small distances toward the target followed by a pause or rest to ensure an accurate completion of the task. Similar observations were described by Jiang et al., who compared a position and velocity control scheme with a nonnegative matrix factorization algorithm [34]. This result designates velocity control as the favored cursor-control scheme for the system. However, we acknowledge that the preferred cur- sor-control technique may depend on the task (e.g., a center-out target-acquisition task using a VH vs an objectmanipulation task using a physical prosthesis) and should be studied further.

The results of Experiment A found no difference in performance between the different electrode arrays tested. The 12-site untargeted electrode array is assumed to measure redundant EMG activity; therefore, a subset of electrodes could measure the equivalent information. Previously, we found that three statistically independent electrodes could be determined by performing a crosscorrelation analysis of the EMG activity measured using a 12-site untargeted surface electrode array for 9 out of 10 subjects tested [35]. For this task, the EMG activity collected by three electrodes provided sufficient information for the controller to decipher user intent with similar accuracy to the 4- and 12-electrode arrays. The 3-site electrode array is thereby the preferred electrode array configuration for this system. This finding has noteworthy clinical implications as well. The number of electrodes necessary to control a myoelectric prosthesis should be 

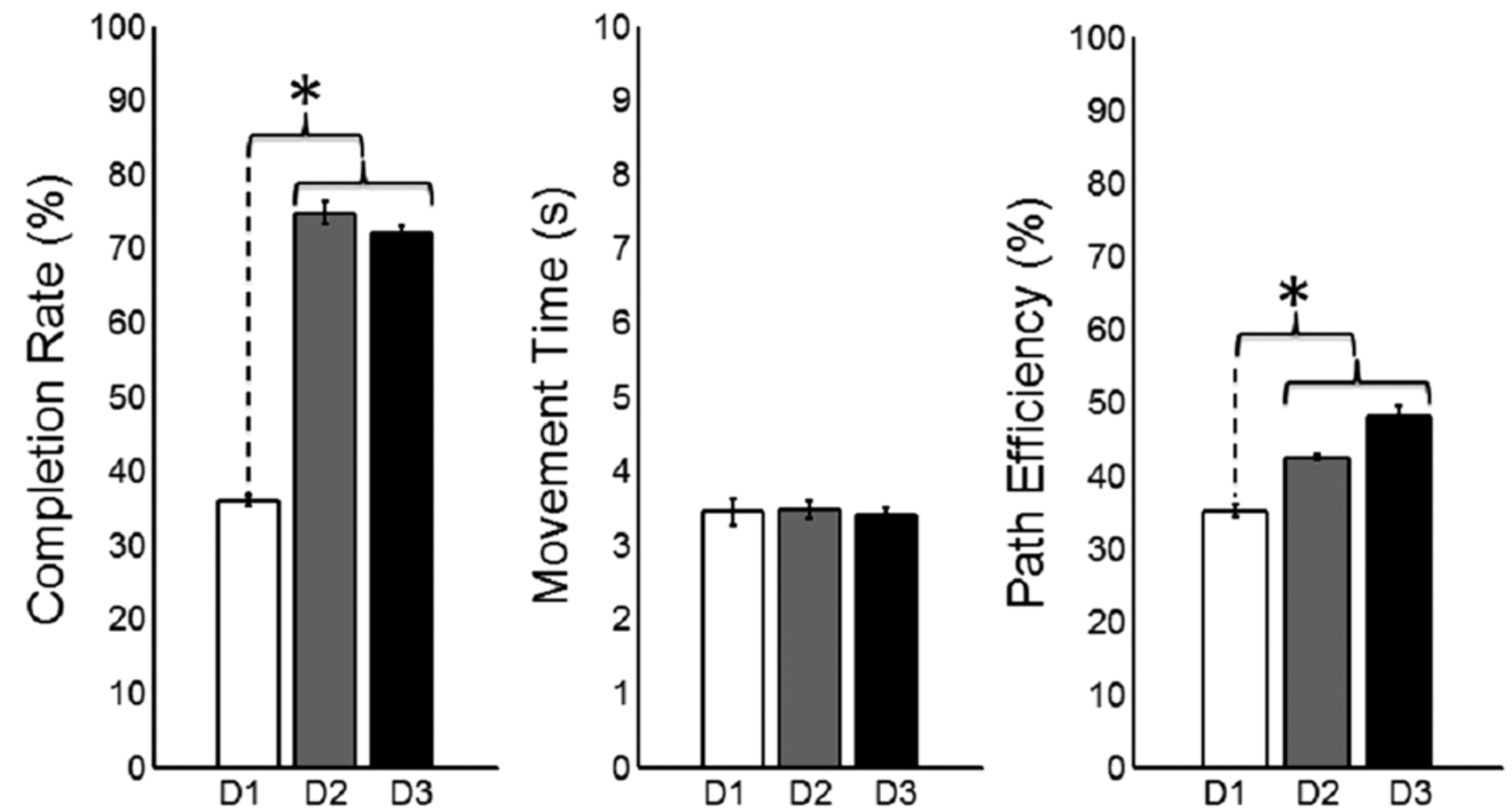

Figure 8.

Experiment B Pretest session performance metrics averaged over subjects for each day (D). ${ }^{*} p<0.05$.

minimized in order to reduce cost and complexity of a prosthetic hand system. We plan to build upon this result by developing a PC domain map for a 2-site electrode array in the future.

\section{Experiment B}

The clinical efficacy of the postural controller was studied using the $\mathrm{T}$ session performance metrics. The average $\mathrm{CR}, \mathrm{MT}$, and $\mathrm{PE}$ during the $\mathrm{T}$ sessions were comparable to many of the current state-of-the-art myo- electric controllers [15-16,26]. Pistohl et al. used a similar control architecture, but did not test a clinically viable system since the control sites were intrinsic to the hand and functional postures were not produced [29]. However, Dalley et al. reported better performance than the work presented here with a state machine architecture [23] using a clinically viable system. The state machine architecture provides a more restrictive environment, within which the user controls the hand posture as compared to

Table 2.

Experiment B performance metrics averaged across days for each subject. Results are shown for target training (Tar), no target training (nTar), and test session (T); $p$-value indicates statistical difference between sessions. Bold $p$-values indicate significant difference.

\begin{tabular}{|c|c|c|c|c|c|c|c|c|c|c|c|c|}
\hline \multirow{2}{*}{ Subject } & \multicolumn{4}{|c|}{ CR (\%) } & \multicolumn{4}{|c|}{ MT (s) } & \multicolumn{4}{|c|}{ PE (\%) } \\
\hline & Tar & nTar & $\mathbf{T}$ & $p$-Value & Tar & nTar & $\mathbf{T}$ & $p$-Value & Tar & nTar & $\mathbf{T}$ & $p$-Value \\
\hline S1 & 82 & 84 & 86 & 0.84 & 4.6 & 4.1 & 4.3 & 0.36 & 52 & 55 & 53 & 0.95 \\
\hline S2 & 98 & 95 & 89 & 0.01 & 3.0 & 3.3 & 3.3 & 0.63 & 38 & 40 & 38 & 0.59 \\
\hline S3 & 99 & 97 & 89 & 0.04 & 2.7 & 2.6 & 2.8 & 0.54 & 46 & 50 & 48 & 0.92 \\
\hline S4 & 91 & 99 & 64 & 0.01 & 3.5 & 3.2 & 3.5 & 0.46 & 39 & 41 & 42 & 0.86 \\
\hline
\end{tabular}


the PC architecture. The state machine limits the user to specific transitions between neighboring postures using a physiologically inappropriate trigger command, while the postural controller allows for direct transitions between all postures in the PC domain without a trigger command. The state machine is a single-dimensional architecture (i.e., a linear arrangement of postures), whereas the PC architecture is a two-dimensional architecture (i.e., a planar arrangement of postures). The added dimension in the PC architecture provides additional freedom while sacrificing performance as compared to the state machine [21]. We plan to study this trade-off in more depth by comparing state machine and PC architectures directly in the future.

The CR for S4 was significantly different compared to the three other subjects $(p=0.01)$. We feel that the EMG acquisition gains and thresholds were poorly tuned (the gains too high and thresholds too low) during D1 for S4. The subject repeatedly overshot the target in the PC domain and lacked the ability to direct the cursor to the target consistently without visual feedback $(<70 \% \mathrm{CR})$. The experimental protocol disallowed retuning of the EMG acquisition system after the first meeting. However, this is only a constraint due to the experimental design, which is to study the robustness of the algorithm across days. In a clinical setting, the EMG acquisition gains and thresholds would be tuned (and retuned) appropriately by a prosthetist when necessary.

The Pt session allowed for an analysis of retention of the ability to use the postural controller across days. The performance of subjects (specifically $\mathrm{CR}$ and $\mathrm{PE}$ ) increased and then persisted after only a single day of training. In other words, a single training session is sufficient for high-level use. This implies that clinical implementation of the postural controller could occur during a single training session between the prosthetist and user.

Another important outcome when comparing performance across days is that performance is not affected by donning or doffing of the myoelectric system. The myoelectric interface was removed completely between days; however, the performance on average increased and then stabilized without retraining or tuning the myoelectric interface. Specifically, this observation differentiates the postural controller from many pattern recognition systems previously developed that show deteriorating performance from donning and doffing [16].

The effect of visual feedback on performance was shown to significantly augment the CR for three out of four subjects. This finding was expected as the visual feedback provided during the training sessions was meant to assist the subjects in completing the task. The information provided by the real-time cursor position enabled the subjects to modulate the muscular activity in order to acquire the target in the PC domain. The MT and PE metrics were unaffected by the various visual feedback paradigms. This suggests that visual feedback assisted in the overall completion of the task (i.e., matching postures) but not in the speed (MT) or precision (PE). However, this study did not test whether subjects would learn more effectively by exploring the PC domain without any visual feedback, which will be tested in future studies.

\section{Novel Aspects}

The postural controller presented here integrates several novel aspects with respect to previous work [2426,31]. Here, the postural map is fully customizable due to the novel derivation of the JAT. Previously, principal component vectors were used to derive the JAT and thereby statically dictated the number and locations of the postures in the PC domain. Now, the locations of the postures are defined by the postural map and implemented using Equation 4. The number and location of the postures in the PC domain is unlimited. The exemplary postural map (Figure 2(d)) depicts a PC domain with seven unique postures (including hand flat); however, additional postures could be added circumferentially between the given postures, radially in front or behind the postures, etc. Furthermore, the PC algorithm allows for the addition of wrist postures to the PC domain; this would provide a system that could control both wrist and hand posture in simultaneous fashion. The freedom provided by this architecture is greater than previous postural controllers due to the advancement in the derivation of the JAT.

The development of the potential field also distinguishes this work. The potential field effectively adds a third dimension to the PC domain. The topography of the PC domain is determined by the design of the feedback controller and the tuning of the proportional and derivative gains. The proportional gain adjusts the "steepness" of the well/wedge, while the derivative gain "flattens" the bottom of the well. An interesting viewpoint is that the potential field transforms the PC domain into a "soft" state machine. The preferred states are located at the areas with lowest potential, and the harshness of the 
states is determined by the depth of the wells/wedges. The exemplary potential field (Figure 2(c)) is a preliminary attempt to design a potential field that augments the performance of the postural controller. Pilot studies indicated the utility of the potential field used here; however, the design of more optimal potential field requires further investigation.

\section{CONCLUSIONS}

The replacement of the human hand after amputation requires solving intensive engineering challenges, including the development of real-time and intuitive MECs. Here, a novel MEC was tested in order to determine key design features and measure performance across multiple days. The use of a velocity cursor-control technique caused performance to increase compared to a position cursor-control technique. Also, the number of surface electrodes used by the subjects $(3,4$, or 12$)$ did not cause a change in performance. These results inform the design of the PC algorithm. The ability to command a virtual hand was measured at a high level and did not degrade across multiple days of testing. This work substantiates the novel algorithm for a postural controller as an effective and robust design for a MEC of multifunction prosthetic hands.

\section{ACKNOWLEDGMENTS}

\author{
Author Contributions: \\ Developed original concept and design of manuscript: R. F. Weir. \\ Conducted original controller development work: J. L. Segil. \\ Helped build experiment apparatus: J. L. Segil. \\ Designed and ran experiments: J. L. Segil. \\ Analyzed resulting data: J. L. Segil. \\ Main contributor to drafting manuscript: J. L. Segil. \\ Critical revision of manuscript: $\mathrm{R}$. F. Weir.
}

Financial Disclosures: The authors have declared that no competing interests exist.

Funding/Support: This material was based on work supported by the Rehabilitation Research \& Development Service of the Department of Veterans Affairs Office of Research and Development (award I01BX007080).

Institutional Review: The Institutional Review Board at the University of Colorado at Boulder reviewed and approved all experimental protocols. Informed consent was obtained from all subjects before each experiment.

Participant Follow-Up: The authors do not plan to inform participants of the publication of this study.

\section{REFERENCES}

1. Moore KL, Dalley AF. Clinically oriented anatomy. 4th ed. Baltimore (MD): Lippincott Williams \& Wilkins; 1999.

2. Belter JT, Segil JL, Dollar AM, Weir RF. Mechanical design and performance specifications of anthropomorphic prosthetic hands: A review. J Rehabil Res Dev. 2013; 50(5):599-618. [PMID:24013909]

3. Battye CK, Nightingale A, Whillis J. The use of myo-electric currents in the operation of prostheses. J Bone Joint Surg Br. 1955;37-B(3):506-10. [PMID:13252063]

4. Dorcas DS, Scott RN. A three-state myo-electric control. Med Biol Eng. 1966;4(4):367-70. [PMID:5964421] http://dx.doi.org/10.1007/BF02476154

5. Parker PA, Stuller J, Scott R. Signal processing for the multistate myoelectric channel. Proc IEEE. 1977;65(5):66274. http://dx.doi.org/10.1109/PROC.1977.10545

6. Childress DS, Weir RF. Control of limb prostheses. Atlas Limb Prosthet. 2004;2:175-98.

7. Biddiss E, Beaton D, Chau T. Consumer design priorities for upper limb prosthetics. Disabil Rehabil Assist Technol. 2007;2(6):346-57. [PMID:19263565] http://dx.doi.org/10.1080/17483100701714733

8. Atkins D, Heard D, Donovan W. Epidemiologic overview of individuals with upper-limb loss and their reported research priorities. J Prosthet Orthot. 1996;8(1):2-11. http://dx.doi.org/10.1097/00008526-199600810-00003

9. Kuiken TA, Li G, Lock BA, Lipschutz RD, Miller LA, Stubblefield KA, Englehart KB. Targeted muscle reinnervation for real-time myoelectric control of multifunction artificial arms. JAMA. 2009;301(6):619-28.

[PMID: 19211469]

http://dx.doi.org/10.1001/jama.2009.116

10. Weir RF, Troyk PR, DeMichele GA, Kerns DA, Schorsch JF, Maas H. Implantable myoelectric sensors (IMESs) for intramuscular electromyogram recording. IEEE Trans Biomed Eng. 2009;56(1):159-71. [PMID:19224729] http://dx.doi.org/10.1109/TBME.2008.2005942

11. Cipriani C, Segil JL, Birdwell JA, ff Weir RF. Dexterous control of a prosthetic hand using fine-wire intramuscular electrodes in targeted extrinsic muscles. IEEE Trans Neural Syst Rehabil Eng. 2014;22(4):828-36. [PMID:24760929] http://dx.doi.org/10.1109/TNSRE.2014.2301234

12. Pasquina PF, Evangelista M, Carvalho AJ, Lockhart J, Griffin S, Nanos G, McKay P, Hansen M, Ipsen D, Vandersea J, Butkus J, Miller M, Murphy I, Hankin D. First-inman demonstration of a fully implanted myoelectric sensors system to control an advanced electromechanical prosthetic hand. J Neurosci Methods. 2015;244:85-93. [PMID:25102286]

13. Hudgins B, Parker P, Scott RN. A new strategy for multifunction myoelectric control. IEEE Trans Biomed Eng. 
1993;40(1):82-94. [PMID:8468080]

http://dx.doi.org/10.1109/10.204774

14. Englehart K, Hudgins B. A robust, real-time control scheme for multifunction myoelectric control. IEEE Trans Biomed Eng. 2003;50(7):848-54. [PMID:12848352] http://dx.doi.org/10.1109/TBME.2003.813539

15. Li G, Schultz AE, Kuiken TA. Quantifying pattern recognition-based myoelectric control of multifunctional transradial prostheses. IEEE Trans Neural Syst Rehabil Eng. 2010;18(2):185-92. [PMID:20071269] http://dx.doi.org/10.1109/TNSRE.2009.2039619

16. Scheme E, Englehart K. Electromyogram pattern recognition for control of powered upper-limb prostheses: State of the art and challenges for clinical use. J Rehabil Res Dev. 2011;48(6):643-59. [PMID:21938652] http://dx.doi.org/10.1682/JRRD.2010.09.0177

17. Young AJ, Smith LH, Rouse EJ, Hargrove LJ. Classification of simultaneous movements using surface EMG pattern recognition. IEEE Trans Biomed Eng. 2013;60(5): 1250-58. [PMID:23247839] http://dx.doi.org/10.1109/TBME.2012.2232293

18. Cipriani C, Controzzi M, Kanitz G, Sassu R. The effects of weight and inertia of the prosthesis on the sensitivity of electromyographic pattern recognition in relax state. J Prosthet Orthot. 2012;24(2):86-92. http://dx.doi.org/10.1097/JPO.0b013e3182524cce

19. Kyberd PJ, Chappell PH. The Southampton Hand: An intelligent myoelectric prosthesis. J Rehabil Res Dev. 1994;31(4):326-34. [PMID:7869280]

20. Kyberd PJ, Holland OE, Chappell PH, Smith S, Tregidgo R, Bagwell PJ, Snaith M. MARCUS: A two degree of freedom hand prosthesis with hierarchical grip control. IEEE Trans Rehabil Eng. 1995;3(1):70-76. http://dx.doi.org/10.1109/86.372895

21. Dalley SA, Varol HA, Goldfarb M. A method for the control of multigrasp myoelectric prosthetic hands. IEEE Trans Neural Syst Rehabil Eng. 2012;20(1):58-67. [PMID:22180515] http://dx.doi.org/10.1109/TNSRE.2011.2175488

22. Cipriani C, Controzzi M, Carrozza MC. The SmartHand transradial prosthesis. J Neuroeng Rehabil. 2011;8(1):29. [PMID:21600048]

http://dx.doi.org/10.1186/1743-0003-8-29

23. Dalley SA, Bennett DA, Goldfarb M. Preliminary functional assessment of a multigrasp myoelectric prosthesis. In: Proceedings of the Engineering in Medicine and Biology Society (EMBC) 2012 Annual International Conference of the IEEE; 2012 Aug 28-Sep 1; San Diego, CA. New York (NY): IEEE; 2012. p. 4172-75.

24. Ciocarlie MT, Allen PK. Hand posture subspaces for dexterous robotic grasping. Int J Robot Res. 2009;28(7):85167. http://dx.doi.org/10.1177/0278364909105606
25. Matrone GC, Cipriani C, Secco EL, Magenes G, Carrozza MC. Principal components analysis based control of a multi-DoF underactuated prosthetic hand. J Neuroeng Rehabil. 2010;7(1):16. [PMID:20416036] http://dx.doi.org/10.1186/1743-0003-7-16

26. Matrone GC, Cipriani C, Carrozza MC, Magenes G. Realtime myoelectric control of a multi-fingered hand prosthesis using principal components analysis. J Neuroeng Rehabil. 2012;9(1):40. [PMID:22703711] http://dx.doi.org/10.1186/1743-0003-9-40

27. Santello M, Flanders M, Soechting JF. Postural hand synergies for tool use. J Neurosci. 1998;18(23):10105-15. [PMID:9822764]

28. de Rugy A, Loeb GE, Carroll TJ. Muscle coordination is habitual rather than optimal. J Neurosci. 2012;32(21): 7384-91. [PMID:22623684] http://dx.doi.org/10.1523/JNEUROSCI.5792-11.2012

29. Pistohl T, Cipriani C, Jackson A, Nazarpour K. Abstract and proportional myoelectric control for multi-fingered hand prostheses. Ann Biomed Eng. 2013;41(12):2687-98. [PMID:23934195] http://dx.doi.org/10.1007/s10439-013-0876-5

30. Radhakrishnan SM, Baker SN, Jackson A. Learning a novel myoelectric-controlled interface task. J Neurophysiol. 2008;100(4):2397-2408. [PMID:18667540] http://dx.doi.org/10.1152/jn.90614.2008

31. Segil JL, Weir RF. Design and validation of a morphing myoelectric hand posture controller based on principal component analysis of human grasping. IEEE Trans Neural Syst Rehabil Eng. 2014;22(2):249-57. [PMID:23649286]

32. Simon AM, Hargrove LJ, Lock BA, Kuiken TA. Target Achievement Control Test: Evaluating real-time myoelectric pattern-recognition control of multifunctional upperlimb prostheses. J Rehabil Res Dev. 2011;48(6):619-27. [PMID:21938650] http://dx.doi.org/10.1682/JRRD.2010.08.0149

33. Salmoni AW, Schmidt RA, Walter CB. Knowledge of results and motor learning: A review and critical reappraisal. Psychol Bull. 1984;95(3):355-86.

[PMID:6399752] http://dx.doi.org/10.1037/0033-2909.95.3.355

34. Jiang N, Rehbaum H, Vujaklija I, Graimann B, Farina D. Intuitive, online, simultaneous, and proportional myoelectric control over two degrees-of-freedom in upper limb amputees. IEEE Trans Neural Syst Rehabil Eng. 2014; 22(3):501-10. [PMID:23996582]

http://dx.doi.org/10.1109/TNSRE.2013.2278411

35. Segil JL, Weir RF. Derivation of optimal surface electrode control sites using untargeted electrode array for myoelectric control of prosthetic hands. Paper presented at: Rocky Mountain American Society of Biomechanics Regional Meeting; 2013 Apr 17-18; Estes Park, CO. 
Submitted for publication June 5, 2014. Accepted in revised form March 12, 2015.

This article and any supplementary material should be cited as follows:

Segil JL, Weir RF. Novel postural control algorithm for control of multifunctional myoelectric prosthetic hands. J
Rehabil Res Dev. 2015;52(4):449-66.

http://dx.doi.org/10.1682/JRRD.2014.05.0134

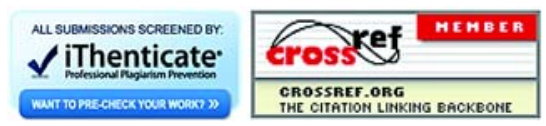


